\title{
Comment on: "Cardiovascular adverse events in patients with chronic lymphocytic leukemia receiving acalabrutinib monotherapy: pooled analysis of 762 patients"
}

We read with great interest the study by Brown et al. ${ }^{1}$ analyzing the cardiovascular events during acalabrutinib treatment within four clinical trials. After a median time of acalabrutinib treatment of 24.9 months in the polled analysis, 38 of 762 chronic lymphocytic leukemia (CLL) patients developed atrial fibrillation (AF) and/or atrial flutter (15 with treatment-naïve and 23 with relapsed/refractory CLL). Rates of AF decreased over time, but were higher among patients with a prior history of arrhythmia as well as in elderly patients.

Bruton's tyrosine kinase (BTK) inhibitors are widely used for the treatment of B-cell malignancies, including CLL. Continuous treatment with the first in class BTK inhibitor, ibrutinib, has been associated with increased incidence of cardiovascular events like $\hat{A} F$, atrial flutter and new onset or worse previous arterial hypertension compared to the control healthy population. ${ }^{2-4}$

The risk of developing ibrutinib-induced AF can be predicted by some AF risk scores developed. ${ }^{3,5-7}$ As reported in Table 1, (i) the Framingham score is based on age, male sex, body mass index, systolic pressure, treatment for hypertension, PR interval, significant murmur, heart failure; ${ }^{3}$ (ii) the Shanafelt score includes age, sex, valvular heart disease and hypertension; ${ }^{6}$ (iii) the Italian score includes age, sex, non-valvular cardiopathy, valvular heart disease, hypo/hyper-thyroidism, chronic lung diseases, diabetes mellitus and previous grade 3-4 infections. ${ }^{5}$ In addition, colleagues from the Mayo clinic analyzed the incidence and management of AF among $290 \mathrm{CLL}$ patients treated with ibrutinib. ${ }^{8}$ After a median time on ibrutinib of 19 months, the authors concluded that all the three scores were able to identify patients with an increased risk to develop AF but "based on lower Akaike information criteria (AIC), the Italian score $(A I C=513)$ was best at predicting the risk of treatment-emergent AF versus the Mayo CLL risk score $(\mathrm{AIC}=524)$ and the Framingham risk score $(\mathrm{AIC}=530)$." ${ }^{8}$

However, in the paper by Brown et al. only the Shanafelt risk score was applied to acalabrutinib-treated patients. ${ }^{1}$

Given the non-inferiority of the Italian AF score, or slightly better prediction accuracy, we would like to suggest stratifying and comparing the cumulative incidence of AF of acalabrutinib-treated patients with both scores.

Recently updated results from the Elevate RR trial have been published. ${ }^{9}$ This pivotal phase III clinical trial compared acalabrutinib versus ibrutinib in relapsed-refractory
Table 1. Atrial fibrillation scores.

\begin{tabular}{|l|c|c|c|}
\hline Variables & $\begin{array}{c}\text { Framingham } \\
\text { score }\end{array}$ & $\begin{array}{c}\text { Shanafelt } \\
\text { score }\end{array}$ & $\begin{array}{c}\text { Italian } \\
\text { score }\end{array}$ \\
\hline Age & $\mathrm{X}$ & $\mathrm{X}$ & $\mathrm{X}$ \\
\hline Male sex & $\mathrm{X}$ & $\mathrm{X}$ & $\mathrm{X}$ \\
\hline Body mass index & $\mathrm{X}$ & & \\
\hline Systolic pressure & $\mathrm{X}$ & & \\
\hline $\begin{array}{l}\text { Treatment for } \\
\text { hypertension }\end{array}$ & $\mathrm{X}$ & & \\
\hline ECG PR interval & $\mathrm{X}$ & & \\
\hline Heart murmur & $\mathrm{X}$ & & \\
\hline Heart failure & & $\mathrm{X}$ & \\
\hline Valvular heart disease & & $\mathrm{X}$ & \\
\hline Hypertension & & $\mathrm{X}$ & \\
\hline Cardiopathy & & $\mathrm{X}$ & \\
\hline Hypo/hyper-thyroidism & & $\mathrm{X}$ & \\
\hline Chronic lung disease & & $\mathrm{X}$ & \\
\hline Diabetes mellitus & & & \\
\hline G3-4 infections & & & \\
\hline
\end{tabular}

ECG: elctrocardiography; PR interval: time from the onset of the $P$ wave to the start of the QRS complex.

patients with high-risk cytogenetics, displaying superimposable efficacy but a better safety profile of acalabrutinib in the management of CLL patients compared to ibrutinib. ${ }^{9}$ In particular AF, hypertension, diarrhea, arthralgia and muscle spasms were less frequent in the acalabrutinib arm, while headache and cough were more common in the acalabrutinib than the ibrutinib arm. ${ }^{9}$ Whether second generation BTK inhibitors, like acalabrutinib, decrease AF rate to all risk classes, in particular to score $\geq 5$, compared to ibrutinib is unknown but is a relevant unmet clinical need.

\section{Authors}

Andrea Visentin and Livio Trentin

Hematology and Clinical Immunology Unit, Department of Medicine, University of Padua, Padova, Italy 


\section{Correspondence:}

ANDREA VISENTIN- andrea.visentin@unipd.it

https://doi.org/10.3324/haematol.2021.280199

Received: October 14, 2021.

Accepted: November 5, 2021.

Prepublished: November 18, 2021.

\section{Disclosures}

No conflicts of interest to disclose.

\section{Contributions}

$\mathrm{AV}$ and $\mathrm{LT}$ reviewed the literature and co-wrote the comment.

\section{References}

1. Brown JR, Byrd JC, Ghia P, et al. Cardiovascular adverse events in patients with chronic lymphocytic leukemia receiving acalabrutinib monotherapy: pooled analysis of 762 patients. Haematologica. 2022;107(6):1335-1346.

2. Ahn IE, Brown JR. Targeting Bruton's tyrosine kinase in CLL. Front Immunol. 2021;12:687458.

3. Wiczer TE, Levine LB, Brumbaugh J, et al. Cumulative incidence, risk factors, and management of atrial fibrillation in patients receiving ibrutinib. Blood Adv. 2017;1(20):1739-1748.

4. Visentin A, Campello E, Scomazzon E, et al. Dabigatran in ibrutinib-treated patients with atrial fibrillation and lymphoproliferative diseases: experience of 4 cases. Hematol Oncol. 2018;36(5):801-803.

5. Visentin A, Deodato M, Mauro FR, et al. A scoring system to predict the risk of atrial fibrillation in chronic lymphocytic leukemia. Hematol Oncol. 2019;37(4):508-512.

6. Shanafelt TD, Parikh SA, Noseworthy PA, et al. Atrial fibrillation in patients with chronic lymphocytic leukemia (CLL). Leuk Lymphoma. 2017;58(7):1630-1639.

7. Brown JR, Moslehi J, O'Brien S, et al. Characterization of atrial fibrillation adverse events reported in ibrutinib randomized controlled registration trials. Haematologica. 2017;102(10): 1796-1805.

8. Archibald WJ, Rabe KG, Kabat BF, et al. Atrial fibrillation in patients with chronic lymphocytic leukemia (CLL) treated with ibrutinib: risk prediction, management, and clinical outcomes. Ann Hematol. 2021;100(1):143-155.

9. Byrd JC, Hillmen P, Ghia P, et al. Acalabrutinib versus ibrutinib in previously treated chronic lymphocytic leukemia: results of the first randomized phase III trial. J Clin Oncol. 2021;39(31):3441-3452. 